\title{
STABILITY IN INTERPOLATION OF FAMILIES OF BANACH SPACES
}

\author{
WEI CAO AND YORAM SAGHER
}

(Communicated by J. Marshall Ash)

\begin{abstract}
Let $D$ be a simply connected domain in the complex plane whose boundary $\Gamma$ is a rectifiable simple closed curve. Let $\{A(\gamma) / \gamma \in \Gamma\}$ and $\{B(\gamma) / \gamma \in \Gamma\}$ be interpolation families of Banach spaces. Let $T$ be a linear operator mapping $A(\gamma)$ continuously into $B(\gamma)$. For $z \in D$ let $T_{z}$ be the restriction of $T$ to the interpolation space $A_{z}$. Then $\left\{z \in D / \operatorname{cod}\left(T_{z}\right)=d<\infty\right.$ and $\left.\operatorname{dim} \operatorname{Ker}\left(T_{z}\right)=0\right\}$ and $\left\{z \in D / \operatorname{dim} \operatorname{Ker}\left(T_{z}\right)=d<\infty\right.$ and $T_{z}$ is onto $\left.B_{z}\right\}$ are open sets.
\end{abstract}

\section{INTRODUCTION}

We begin with a brief review of the theory of interpolation for analytic families of Banach spaces. Throughout the paper, $D$ will denote a simply connected domain in the complex plane whose boundary $\Gamma$ is a rectifiable simple closed curve.

Definition 1.1. An interpolation family of Banach spaces on $\Gamma$ is a collection $\{A(\gamma) \mid \gamma \in \Gamma\}$, of Banach spaces which satisfies:

(1) Every $A(\gamma)$ is continuously embedded in a common Banach space $\mathscr{U}$ which is called a containing space for $\{A(\gamma) \mid \gamma \in \Gamma\}$.

(2) For every $a \in \cap_{\gamma \in \Gamma} A(\gamma),\|a\|_{A(\gamma)}$ is a measurable function on $\Gamma$ with respect to $d P_{z}$, where $z \in D$ and $d P_{z}$ is a harmonic measure on $\Gamma$ with respect to $z$.

(3) Let $\mathscr{A}=\left\{a \in \cap_{\gamma \in \Gamma} A(\gamma) \mid \int_{\Gamma} \log ^{+}\|a\|_{A_{\gamma}} d P_{z}(\gamma)<\infty\right\}$, where $\log ^{+} x=$ $\max (\log x, 0) . \mathscr{A}$ is a linear space which is called the $\log$-intersection space for $\{A(\gamma) \mid \gamma \in \Gamma\}$. We assume that $\mathscr{A}$ is dense in each $A(\gamma)$ and that there exists a measurable function $\psi$ on $\Gamma$ which satisfies $\int_{\Gamma} \log ^{+} \psi(\gamma) d P_{z}<\infty$ such that $\|a\|_{u} \leq \psi(\gamma)\|a\|_{A(\gamma)}$ for all $a \in \mathscr{A}$ and $\gamma \in \Gamma$.

We shall denote the spaces $A(\gamma)$ by $A_{\gamma}$ and their norms by $\|\cdot\|_{\gamma}$.

Received by the editors October 4, 1989 and, in revised form, February 2, 1990.

1980 Mathematics Subject Classification (1985 Revision). Primary 47A53. 
We denote by $N^{+}(D)$ the class of all functions $f(z)$ analytic on $D$ such that $f(\phi(w))$ belongs to the class $N^{+}=N^{+}(\Delta)$, where $\Delta=\{w|| w \mid<1\}$ and $\phi$ is a conformal map from $D$ to $\Delta$, see [7]. Thus $N^{+}(D)$ is closed under pointwise addition and multiplication and each $f(z) \in N^{+}(D)$ possesses a.e. non-tangential limits on $\Gamma$. If these non-tangential limits are essentially bounded on $\Gamma$, then $f \in H^{\infty}(D)$, the space of bounded analytic functions on $D$. A function $f \in N^{+}(D)$ is termed an outer function in $N^{+}(D)$, if $f(\phi(w))$ is an outer function in $N^{+}(\Delta)$.

Let $\mathscr{G}(A(\cdot), \Gamma)=\mathscr{G}(\mathscr{A})=\mathscr{G}$ be the space of all functions of the form $g(z)=\Sigma_{j=1}^{n} \varphi_{j}(z) a_{j}$, where $a_{j} \in \mathscr{A}$ and $\varphi_{j} \in N^{+}(D)$, and such that

$$
\|g(\cdot)\|_{\mathscr{G}}=\operatorname{ess} \sup _{\gamma \in \Gamma}\|g(\gamma)\|_{\gamma}<\infty .
$$

Note that, in general, $\left(\mathscr{G},\|\cdot\|_{\mathscr{G}}\right)$ is not complete.

Definition 1.2. For $z \in D$ and $a \in \mathscr{A}$, define

$$
\|a\|_{A\{z\}}=\inf \left\{\|g\|_{\mathscr{g}} \mid g \in \mathscr{G}, g(z)=a\right\} .
$$

The interpolation space $\left\{A_{\gamma}\right\}\{z\}=A\{z\}$ is defined to be the completion in $\mathscr{U}$ of $\left(\mathscr{A},\|\cdot\|_{A\{z\}}\right)$.

In most applications the family of dual spaces $\left\{A_{\gamma}^{*}\right\}$ is itself an interpolation family and $(A\{z\})^{*}=\left\{A_{\gamma}^{*}\right\}\{z\}$. In this paper we shall assume that the above duality result holds.

We consider in this paper linear operators $T$ mapping $\mathscr{A}$ into $\cap_{\gamma \in \Gamma} B(\gamma)$, with $\|T a\|_{B(\gamma)} \leq M(\gamma)\|a\|_{A(\gamma)}$ for all $a \in \mathscr{A}$ and $\gamma \in \Gamma$, where $\{A(\gamma) \mid \gamma \in \Gamma\}$ and $\{B(\gamma) \mid \gamma \in \Gamma\}$ are interpolation families on $\Gamma$ and

$$
\int_{\Gamma}|\log M(\gamma)| d P_{z}(\gamma)<\infty
$$

We will denote the restriction of $T$ to $A\{z\}$ by $T_{z}$. If $\operatorname{ker} T_{z}=\{0\}$, denote by $T_{z}^{-1}$ the inverse of $T_{z}$, i.e., $T_{z}^{-1}$ is defined on $T_{z} A\{z\}$. If $T_{z} A\{z\}$ has finite codimension, by the open mapping theorem, $T_{z} A\{z\}$ is closed and $T_{z}^{-1}$ is a bounded linear operator.

The question of the stability of Fredholm property when one changes the parameters which determine the interpolation space has been considered by several authors $[1,4,9,12]$ in the context of interpolation of two spaces and by Vignati and Vignati [11] in the case of families of Banach spaces. We consider families of Banach spaces and prove that, if $\operatorname{ker} T_{s}=\{0\}$ and $\operatorname{codim} T_{s}=d<$ $\infty$, then there exists a ball $B$ centered at $s$ such that $z \in B$ implies that $\operatorname{ker} T_{z}=\{0\}$ and $\operatorname{codim} T_{z}=d$. This generalizes the result in [11], where the theorem is proved in the case $d=0$.

Definition 1.3. Suppose $U(A)$ is a subset of $\mathscr{G}(\mathscr{A})$. Let

$$
U_{s}=\{a \in A\{s\} \mid \exists f \in U(A) \text { such that } f(s)=a\} .
$$


Define

$$
\|a\|_{U_{s}(A)}=\inf \left\{\|f\|_{\mathscr{G}} \mid f(s)=a, f \in U(A)\right\} .
$$

Clearly $\|a\|_{A\{s\}} \leq\|a\|_{U_{s}(A)}$ for all $a \in U_{s}$.

Let $U_{\{s\}}$ be a completion in $A\{s\}$ of $\left\{U_{s},\|\cdot\|_{U_{s}}\right\}$. Clearly $U_{\{s\}} \subset A\{s\}$. If also $\|a\|_{U_{s}(A)}<k\|a\|_{A\{s\}}$, for some $k$ and all $a \in U_{s}$ with $a \neq 0$, we call $U(A)$ an $s$-Calderón subset (with constant $k$ ). If $U(A)$ is an $s$-Calderón subset and a linear subspace of $\mathscr{G}(\mathscr{A})$, we will say that it is an $s$-Calderón subspace.

Clearly $U(A)$ is an $s$-Calderón subspace for some $k$ if and only if $U_{\{s\}}$ is a closed subspace of $A\{s\}$.

An important tool in handling finite dimensional subspaces is Auerbach's lemma:

Lemma 1.4. Let $M$ be a Banach space with dimension $d<\infty$. Then there exist $\left\{e_{1}, \ldots, e_{d}\right\} \subset M$ and $\left\{f_{1}, \ldots, f_{d}\right\} \subset M^{*}$ such that

(1) $\left\|e_{i}\right\|_{M}=1$ and $\left\|f_{i}\right\|_{M^{*}}=1, i=1, \ldots, d$.

(2) $f_{i}\left(e_{j}\right)=\delta_{i j}$

For a proof see [8].

Given a finite set of vectors $\left\{e_{1}, \ldots, e_{d}\right\}$ in a Banach space $A$, we define

$$
b\left(e_{1}, \ldots, e_{d}\right)=\min \left\{\left\|\sum_{i=1}^{d} c_{i} e_{i}\right\|_{A}|\max | c_{i} \mid=1\right\} .
$$

Clearly $\left\{e_{1}, \ldots, e_{d}\right\}$ are independent if and only if $b\left(e_{1}, \ldots, e_{d}\right)>0$. Given $\sum_{i=1}^{d} c_{i} e_{i}$, let $m=\max \left\{\left|c_{i}\right|\right\}$. Then $\left\|\sum_{i=1}^{d} c_{i} e_{i}\right\|_{A}=m\left\|\sum_{i=1}^{d} c_{i} e_{i} / m\right\|_{A} \geq$ $m b\left(e_{1}, \ldots, e_{d}\right)$, so that $\left|c_{i}\right| \leq\left\|\sum_{i=1}^{d} c_{i} e_{i}\right\|_{A} / b\left(e_{1}, \ldots, e_{d}\right)$.

Lemma 1.5. Let $M$ be a Banach space with dimension $d<\infty$. Then $\left\{e_{1}, \ldots\right.$, $\left.e_{d}\right\}$ can be found so that $\left\|e_{i}\right\|_{M}=1$, and $b\left(e_{1}, \ldots, e_{d}\right)=1$.

For a proof, see [4].

Lemma 1.6. Let $E$ be a closed subspace of $A\{s\}$ with finite codimension $d$. Then there exists $\left\{v_{i}, i=1, \ldots, d\right\} \subset \mathscr{G}(\mathscr{A})$, such that $A\{s\}=E \oplus \operatorname{span}\left\{v_{i}(s)\right\}$.

Proof. Since $E$ has finite codimension $d, A\{s\}=E \oplus M$ with $\operatorname{dim} M=d$. Assume that $\left\{e_{i}, 1 \leq i \leq d\right\}$ forms a basis for $M$ with $\left\|e_{i}\right\|_{A\{s\}}=1$ and $b\left(e_{1}, \ldots, e_{d}\right)=1$. Let

$$
\rho=\rho\left(e_{1}, \ldots, e_{d} ; E\right)=\inf \left\{\left\|\sum_{i=1}^{d} c_{i} e_{i}-x\right\|_{A\{s\}}|\max | c_{i} \mid=1, x \in E\right\} .
$$

Clearly $\rho \leq 1$. If $\rho>0$ then $E \cap M=\{0\}$ and $\left\{e_{i}\right\}$ are independent. Since $E$ is a closed subspace and $M$ has finite dimension, then $E \cap M=\{0\}$ implies $\rho>0$. 
Since $\{v(s), v \in \mathscr{G}(\mathscr{A})\}$ is dense in $A\{s\}$, we can find $\left\{v_{i}, i=1, \ldots, d\right\} \subset$ $\mathscr{G}(\mathscr{A})$ with $\left\|v_{i}(s)-e_{i}\right\|_{A\{s\}}<\rho / 2 d$. Now suppose $\max \left\{\left|c_{i}\right|\right\}=1$ and let $x \in E$; we have

$$
\left\|x-\sum_{i=1}^{d} c_{i} v_{i}(s)\right\|_{A\{s\}} \geq\left\|x-\sum_{i=1}^{d} c_{i} e_{i}\right\|_{A\{s\}}-\left\|\sum_{i=1}^{d} c_{i}\left(v_{i}(s)-e_{i}\right)\right\|_{A\{s\}}>\rho / 2 .
$$

Taking infimum we get $\rho\left(v_{1}(s), \ldots, v_{d}(s) ; E\right) \geq \rho / 2>0$. Hence $\left\{v_{i}(s)\right\}$ are linearly independent and $A\{s\}=E \oplus \operatorname{span}\left\{v_{i}(s)\right\}$.

The function $\rho$ introduced in the last proof is, of course, a generalization of the function $b\left(e_{1}, \ldots, e_{d}\right)$. Another application for the function $\rho$ is the following lemma.

Lemma 1.7. Let $E$ be a closed subspace of a Banach space $B$, and let $M$ be a finite dimensional subspace of $B$. Then $E+M$ is closed.

Proof. We may assume $E \cap M=\{0\}$. Let $\left\{e_{1}, \ldots, e_{d}\right\}$ be a basis for $M$. Then $\rho=\rho\left(e_{1}, \ldots, e_{d} ; E\right)>0$. Let $x_{n} \in E+M$, and $x_{n} \rightarrow x_{0} \in B$. $x_{n}=y_{n}+\sum_{i=1}^{d} c_{i}^{n} e_{i}$. Let $M_{n}=\max \left\{\left|c_{i}^{n}\right| \mid i=1, \ldots, d\right\}$.

Given $\varepsilon>0$, let $N$ be so that, for all $n \geq N$, $\left\|x_{n}\right\| \leq(1+\varepsilon)\left\|x_{0}\right\|$. Then $\rho \leq\left\|\sum_{i=1}^{d} c_{i}^{n} e_{i}+y_{n}\right\| / M_{n} \leq(1+\varepsilon)\left\|x_{0}\right\| / M_{n}$, and $M_{n} \leq(1+\varepsilon)\left\|x_{0}\right\| / \rho$. Thus, $\lim \sup M_{n} \leq\left\|x_{0}\right\| / \rho$ and $\left\{\sum_{i=1}^{d} c_{i}^{n} e_{i}\right\}$ is compact. Denote $\sum_{i=1}^{d} c_{i}^{n} e_{i}=z_{n}$. Let $z_{n_{k}} \rightarrow z \in M$; then $y_{n_{k}}=x_{n_{k}}-z_{n_{k}} \rightarrow x_{0}-z$ and, since $E$ is closed, $x_{0}-z \in E$.

We will also need the following well-known result due to Szegö [10].

Lemma 1.8. Let $f(\gamma)$ be a positive $d P_{z}$ measurable function on $\Gamma$ such that

$$
\int_{\Gamma}|\log f(\gamma)| d P_{z}(\gamma)<\infty
$$

for some (and thus every) $z \in D$. Then there exists a non-vanishing outer function $G(z)$ in $N^{+}(D)$ whose a.e. non-tangential limits $G(\gamma)=\lim _{z \rightarrow \gamma} G(z)$ satisfy $|G(\gamma)| f(\gamma)=1$ for a.e. $\gamma \in \Gamma$.

\section{Stability}

An invariant form of Schwarz's lemma states that if $f(z)$ is analytic and $|f(z)|<1$ on $\Delta$, then for any $z, \zeta \in \Delta$,

$$
\left|\frac{f(z)-f(\zeta)}{1-\overline{f(z)} f(\zeta)}\right| \leq\left|\frac{z-\zeta}{1-\bar{z} \zeta}\right| .
$$

This implies that if $z_{0}, \zeta_{0}, z \in \Delta$ and if we denote

$$
m_{z_{0}}(z)=\frac{z-z_{0}}{1-\overline{z_{0}} z} e^{i \alpha},
$$


then

$$
\left|\frac{m_{z_{0}}(z)-m_{z_{0}}(\zeta)}{1-\overline{m_{z_{0}}(z)} m_{z_{0}}(\zeta)}\right|=\left|\frac{z-\zeta}{1-\bar{z} \zeta}\right| .
$$

This, in turn, implies that if $z, \zeta \in D$ and $d$ is a conformal map of $D$ onto $\Delta$, then

does not depend on $d$.

$$
q(z, \zeta)=\left|\frac{d(z)-d(\zeta)}{1-\overline{d(z)} d(\zeta)}\right|
$$

The invariant form of Schwarz's lemma also implies the following lemma.

Lemma 2.1. Let $\phi$ be analytic on $D,|\phi(z)| \leq M$. If $s, z \in D$, then

$$
|\phi(z)| \geq M \frac{|\phi(s)|-M q(z, s)}{M-|\phi(s)| q(z, s)} .
$$

For a proof see [9]. Of course, since $q(z, s)<1$, the inequality above implies $|\phi(z)| \geq|\phi(s)|-q(z, s) M$.

Lemma 2.2. If $f \in \mathscr{G}(\mathscr{A})$ and $z, s \in D$, then

$$
\|f(z)\|_{A\{z\}} \geq\|f(s)\|_{A\{s\}}-q(z, s)\|f\|_{\mathscr{G}} .
$$

Proof. Let us fix $s \in D$. Since $f(s) \in A\{s\}$, it is possible to find $\psi \in A^{*}\{s\}$ such that $\|\psi\|_{A^{*}\{s\}}=1$ and $\psi(f(s))=\|f(s)\|_{A\{s\}}$.

Given $\varepsilon>0$, we can find $G \in \mathscr{G}\left(A^{*}\right)$ such that $\|G(s)-\psi\|_{A^{*}\{s\}}<\varepsilon$ and $\|G\|_{\mathscr{G}\left(\mathscr{A}^{*}\right)}<1+\varepsilon$. Thus $\phi(z)=G(z)(f(z))$ is analytic in $D, \phi \in H^{\infty}(D)$, and

$$
|\phi(z)| \leq(1+\varepsilon)\|f(z)\|_{A\{z\}} \leq(1+\varepsilon)\|f\|_{\mathscr{G}} .
$$

By Lemma 2.1, we have

$$
\begin{aligned}
\|f(z)\|_{A\{z\}} & \geq \frac{|\phi(z)|}{1+\varepsilon} \geq \frac{|\phi(s)|}{1+\varepsilon}-q(z, s)\|f\|_{\mathscr{G}} \\
& \geq \frac{1-\varepsilon}{1+\varepsilon}\|f(s)\|_{A\{s\}}-q(z, s)\|f\|_{\mathscr{G}} .
\end{aligned}
$$

Letting $\varepsilon \rightarrow 0$, we get the theorem.

Theorem 2.3. Let $\left\{v_{1}, \ldots, v_{d}\right\} \subset \mathscr{G}(\mathscr{A})$, and assume that $\left\{v_{i}(s)\right\}$ are independent. Denote $b_{z}=b\left(v_{1}(z), \ldots, v_{d}(z)\right)$. Then there exists $\delta>0$ so that, for all $|z-s|<\delta, b_{z}>b_{s} / 2$. Consequently, if we denote by $U$ the span of $\left\{v_{i}\right\}$ in $\mathscr{G}(\mathscr{A})$ then, for these $z, \operatorname{dim}\left(U_{\{z\}}\right)=d$.

Proof. Without loss of generality, we can assume that $\left\|v_{i}\right\|_{\mathscr{G}}=1$. As we consider $v_{i}(z)$ in $A\{z\}, b_{z}$ will be controlled by inequality (2.1), which will prove the theorem:

$$
\left\|\sum_{i=1}^{d} c_{i} v_{i}(z)\right\|_{A\{z\}} \geq\left\|\sum_{i=1}^{d} c_{i} v_{i}(s)\right\|_{A\{s\}}-q(z, s)\left\|\sum_{i=1}^{d} c_{i} v_{i}\right\|_{\mathscr{G}} .
$$


Since $\left\{v_{i}(s)\right\}$ are independent, we have $b_{s}>0$. Suppose $\max \left\{\left|c_{i}\right|\right\}=1$; then

$$
b_{s} \leq\left\|\sum_{i=1}^{d} c_{i} v_{i}(s)\right\|_{A\{s\}} \text { and }\left\|\sum_{i=1}^{d} c_{i} v_{i}\right\|_{\mathscr{G}} \leq \sum_{i=1}^{d}\left|c_{i}\right| \cdot\left\|v_{i}\right\|_{\mathscr{G}} \leq d .
$$

Hence,

$$
\left\|\sum_{i=1}^{d} c_{i} v_{i}(z)\right\|_{A\{z\}} \geq b_{s}-d q(z, s) .
$$

We can choose $\delta$ small enough such that $|z-s|<\delta$ implies

$$
b_{s}-d q(z, s)>b_{s} / 2 \text {. }
$$

It follows that $b_{z}>b_{s} / 2$. Therefore, if $|z-s|<\delta,\left\{v_{i}(z)\right\}$ are independent and $\operatorname{dim}\left(U_{\{z\}}\right)=d$.

Theorem 2.4. Let $\left\{v_{1}, \ldots, v_{d}\right\} \subset \mathscr{G}(\mathscr{A})$, and assume that $\left\{v_{i}(s)\right\}$ are independent. Denote by $U$ the span of $\left\{v_{i}\right\}$ in $\mathscr{G}(\mathscr{A})$. Then $\exists \delta>0$, such that $|z-s|<\delta$ implies $U$ is a $z$-Calderón space with a uniform constant $k$.

Proof. Without loss of generality, we can assume that $\left\|v_{i}(s)\right\|_{A\{s\}}=1$. Let $m=\max \left\{\left\|v_{i}\right\|_{\mathscr{G}}\right\}$ and $b_{z}=b\left(v_{1}(z), \ldots, v_{d}(z)\right)$. Then, as we have seen before, if $b_{z}>0$, we have

$$
\left|c_{i}\right| \leq \frac{\left\|\sum_{i=1}^{d} c_{i} v_{i}(z)\right\|_{A\{z\}}}{b_{z}}
$$

so that

$$
\left\|\sum_{i=1}^{d} c_{i} v_{i}\right\|_{\mathscr{G}} \leq \frac{m d}{b_{z}}\left\|\sum_{i=1}^{d} c_{i} v_{i}(z)\right\|_{A\{z\}} .
$$

Since $b_{s}>0$, by Theorem $2.3, \exists \delta>0$ such that $|z-s|<\delta$ implies that $b_{z}>b_{s} / 2$ and $U$ is a $z$-Calderón subspace with constant $2 m d / b_{s}$.

Theorem 2.5. Let $U(A), V(A)$ be two z-Calderón subspaces with constant $k$, for all $z$ in a ball $S$ which is contained in $D$. Then the function

$$
\chi_{z}=\inf \left\{d\left(f(z), U_{\{z\}}\right) \mid f \in V(A) \text { and }\|f(z)\|_{A\{z\}}=1\right\}
$$

is continuous in $S$.

Proof. Given $0<\varepsilon<1$ and $s \in S$, there are $v \in V(A), u \in U(A)$ so that

$$
\begin{gathered}
1=\|v(s)\|_{A\{s\}} \leq\|v\|_{\mathscr{G}} \leq k\|v(s)\|_{A\{s\}}=k \\
\|u(s)-v(s)\|_{A\{s\}}<\chi_{s}+\varepsilon / 2
\end{gathered}
$$

with

Therefore

$$
\|u\|_{\mathscr{G}} \leq k\|u(s)\|_{A\{s\}}
$$

$$
\|u\|_{\mathscr{G}} \leq k\left(\|u(s)-v(s)\|_{A\{s\}}+\|v(s)\|_{A\{s\}}\right)<k\left(\chi_{s}+2\right) .
$$


Clearly we have

By Lemma 2.2, we have

$$
\chi_{z} \leq \frac{\|u(z)-v(z)\|_{A\{z\}}}{\|v(z)\|_{A\{z\}}}
$$

$$
\|v(z)\|_{A\{z\}} \geq\|v(s)\|_{A\{s\}}-q(z, s)\|v\|_{\mathscr{G}}=1-q(z, s)\|v\|_{\mathscr{G}} .
$$

Hence we can choose $\eta>0$ small enough such thai $|z-s|<\eta$ implies both $\|v(z)\|_{z}^{-1}<1+\varepsilon$ and $z \in S$. Again, invoking Lemma 2.2, we have

$$
\begin{aligned}
\|u(z)-v(z)\|_{A\{z\}} & \leq\|u(s)-v(s)\|_{A\{s\}}+q(z, s)\|u-v\|_{\mathscr{G}} \\
& \leq\|u(s)-v(s)\|_{A\{s\}}+q(z, s)\left(\|u\|_{\mathscr{G}}+\|v\|_{\mathscr{G}}\right) \\
& <\left(\chi_{s}+\varepsilon / 2\right)+q(z, s)\left(\chi_{s}+3\right) k .
\end{aligned}
$$

Therefore, if necessary, choosing a smaller $\eta>0$, we have that, for $|z-s|<\eta$,

$$
\chi_{z} \leq \frac{\|u(z)-v(z)\|_{A\{z\}}}{\|v(z)\|_{A\{z\}}} \leq(1+\varepsilon)\left(\chi_{s}+\varepsilon\right) .
$$

Similarly $\chi_{s} \leq(1+\varepsilon)\left(\chi_{z}+\varepsilon\right)$ in $|z-s|<\eta_{1}$, and hence $\chi_{z}$ is continuous in $S$.

Note that when $V(A)$ is finite dimensional, as it is in the proof below, $\chi_{z}$ is equivalent to the function $\rho$ defined in the proof of Lemma 1.6.

Theorem 2.6. Let $U$ be a $z$-Calderón subspace with constant $k$, for all $z$ in a ball $S$ contained in $D$. Assume that, for an $s \in S, U_{\{s\}}$ has codimension $d<\infty$. Then there exists $\delta>0$, so that, for all $z$ with $|z-s|<\delta$, we have $\operatorname{codim} U_{\{z\}}=d$.

Proof. By Lemma 1.6, there exist $\left\{v_{i} \mid i=1, \ldots, d\right\} \subset \mathscr{G}(\mathscr{A})$ so that $\left\{v_{i}(s)\right\}$ forms a basis for $M_{s}$ and $A\{s\}=U_{\{s\}} \oplus M_{s}$. We can assume $\left\|v_{i}\right\|_{\mathscr{G}}=1$. Denote by $M_{z}$ the space spanned by $\left\{v_{i}(z)\right\}$. Since $A\{s\}=U_{\{s\}} \oplus M_{s}$ and $U_{\{s\}}, M_{s}$ are closed, there exists a projection $P$ of $A\{s\}$ onto $U_{\{s\}}$ with $\operatorname{ker} P=M_{s}$, and $\|P\|<\infty$.

Since $U$ is a $z$-Calderón subspace, $U_{\{z\}}$ is a closed subspace of $A\{z\}$. By Lemma 1.7 , since $M_{z}$ is finite-dimensional, $U_{\{z\}}+M_{z}$ is closed. Hence, if $A\{z\} \neq U_{\{z\}}+M_{z}$ then there exists $e \in \mathscr{G}(\mathscr{A})$, with $\|e(z)\|_{A\{z\}}=1$ and $1 \leq\|e\|_{\mathscr{G}(\mathscr{A})}<2$, such that $\operatorname{dist}\left(e(z), U_{\{z\}}+M_{z}\right)>2 / 3$.

Consider $e(s) \in A\{s\}$. We have $e(s)=y_{s}+z_{s}$, where $y_{s}=\operatorname{Pe}(s) \in U_{\{s\}}$, $z_{s}=(I-P) e(s)=\sum_{i=1}^{d} c_{i} v_{i}(s) \in M_{s}$. We have

$$
\begin{gathered}
\left\|y_{s}\right\|_{A\{s\}} \leq\|P\|\|e(s)\|_{A\{s\}}<2\|P\| \\
\left\|z_{s}\right\|_{A\{s\}} \leq(\|P\|+1)\|e(s)\|_{A\{s\}}<2(\|P\|+1) .
\end{gathered}
$$

Since $U$ is $s$-Calderón, there exists $f \in U(A)$ such that $\left\|f(s)-y_{s}\right\|_{A\{s\}} \leq$ $\min \left\{1 / 3,\left\|y_{s}\right\|_{A\{s\}}\right\}$ and

$$
\|f\|_{\mathscr{G}} \leq k\|f(s)\|_{A\{s\}} \leq 2 k\left\|y_{s}\right\|_{A\{s\}}<4 k\|P\| \text {. }
$$


On the other hand we have, denoting $b=b\left(v_{1}(s), \ldots, v_{d}(s)\right)$,

$$
\left|c_{i}\right| \leq \frac{\left\|\sum_{i=1}^{d} c_{i} v_{i}(s)\right\|_{A\{s\}}}{b}=\frac{\left\|z_{s}\right\|_{A\{s\}}}{b}<2 \frac{\|P\|+1}{b} .
$$

It follows that

$$
\left\|\sum_{i=1}^{d} c_{i} v_{i}\right\|_{\mathscr{G}} \leq \sum_{i=1}^{d}\left|c_{i}\right|\left\|v_{i}\right\|_{\mathscr{G}} \leq \frac{2 d(\|P\|+1)}{b} .
$$

Let $g=f+\sum_{i=1}^{d} c_{i} v_{i}$. Clearly $\|g(s)-e(s)\|_{A\{s\}}<1 / 3$ and

$$
\begin{aligned}
\|g-e\|_{\mathscr{G}} & \leq\|f\|_{\mathscr{G}}+\left\|\sum_{i=1}^{d} c_{i} v_{i}\right\|_{\mathscr{G}}+\|e\|_{\mathscr{G}} \\
& \leq 4 k\|P\|+\frac{2 d(\|P\|+1)}{b}+2 .
\end{aligned}
$$

Choose $\delta$ such that $|z-s|<\delta$ implies

$$
q(z, s)\left[4 k\|P\|+\frac{2 d(\|P\|+1)}{b}+2\right]<1 / 3
$$

Then, by Lemma 2.2,

$$
\begin{aligned}
\|e(z)-g(z)\|_{A\{z\}} & \leq\|e(s)-g(s)\|_{A\{s\}}+q(z, s)\|e-g\|_{\mathscr{G}} \\
& \leq 1 / 3+q(z, s)\left[4 k\|P\|+\frac{2 d(\|P\|+1)}{b}+2\right]<2 / 3
\end{aligned}
$$

which contradicts $\operatorname{dist}\left(e(z), U_{\{z\}}+M_{z}\right)>2 / 3$. Therefore $U_{\{z\}}+M_{z}=A\{z\}$.

Since $A\{s\}=U_{\{s\}} \oplus M_{s}, U_{\{s\}}$ is closed and $M_{s}$ has finite dimension, we have $\chi_{s}>0$. By Theorem 2.5, there exists $\delta>0$ such that $|z-s|<\delta$ implies $\chi_{z}>0$, which implies $U_{\{z\}} \cap M_{z}=\{0\}$. Hence the codimension of $U_{\{z\}}$ is $d$.

Theorem 2.7. If $\operatorname{ker} T_{s}=\{0\}$ and $\operatorname{codim}\left(T_{s} A\{s\}\right)=d<\infty$, then there exists $\delta>0$, so that, for all $|z-s|<\delta, \operatorname{ker} T_{z}=\{0\}$ and $\operatorname{codim} T_{z} A\{z\}=d$.

Proof. The control over $\operatorname{ker} T_{z}$ is achieved by considering the following function:

$$
r\left(T_{z}\right)=\inf \left\{\|T a\|_{B\{z\}} \mid\|a\|_{A\{z\}}=1\right\} .
$$

Clearly if $r\left(T_{z}\right)>0, \operatorname{ker} T_{z}=\{0\}$ and the range of $T_{z}$ is closed. Conversely, if the range of $T_{z}$ is closed and $\operatorname{ker} T_{z}=\{0\}$ then, by the open mapping theorem, $r\left(T_{z}\right)=\left\|T_{z}^{-1}\right\|^{-1}>0$. Since $\operatorname{codim} T_{s}=d<\infty$, the range of $T_{s}$ is closed, and, since $\operatorname{ker} T_{s}=\{0\}$, we have $r\left(T_{s}\right)>0$. Theorem 2.3 in [11] says that $r\left(T_{z}\right)>r\left(T_{s}\right) / 2>0$ for all $z$ which satisfy $|z-s|<\delta$, some $\delta>0$.

Since $\|T\|_{A(\gamma) \rightarrow B(\gamma)} \leq M(\gamma)$ with $\int_{\Gamma}|\log M(\gamma)| d P_{z}(\gamma)<\infty$, by Lemma 1.8, there exists an outer function $G(\cdot)$ such that

$$
|G(\gamma) M(\gamma)|=1 \text { for a.e. } \gamma \in \Gamma \text {. }
$$


We claim that $G(\cdot) T \mathscr{G}(\mathscr{A})$ is a $z$-Calderón subspace of $\mathscr{G}(\mathscr{B})$ with constant $c$, for all $z$ such that $|z-s|<\delta$, where

$$
c=4\left\|T_{s}^{-1}\right\| \sup _{\eta}\left\{\exp \left(\int \log M(\gamma) d P_{\eta}(\gamma)\right)|| \eta-s \mid<\delta\right\} .
$$

In fact, let $y \in G(\cdot) T \mathscr{G}(\mathscr{A})_{z}$. Then $y \in T_{z} \mathscr{A}$, and so there exists $x \in \mathscr{A}$ such that $T x=y$ and $\|x\|_{A\{z\}} \leq\left\|T_{z}^{-1}\right\|\|y\|_{B\{z\}}$. We choose $g \in \mathscr{G}(\mathscr{A})$ such that $g(z)=x$ and

$$
\|g\|_{\mathscr{G}(\mathscr{A})} \leq 2\|x\|_{A\{z\}} \leq 2\left\|T_{z}^{-1}\right\|\|y\|_{B\{z\}} .
$$

Then $g(\zeta) / G(z) \in \mathscr{G}(\mathscr{A})$ and $H(\zeta)=G(\zeta) T(g(\zeta) / G(z)) \in G(\cdot) T \mathscr{G}(\mathscr{A})$. Clearly $H(z)=y$, and, by (2.4), (2.5),

$$
\|H\|_{\mathscr{G}(\mathscr{B})} \leq \frac{2\left\|T_{z}^{-1}\right\|\|y\|_{B\{z\}}}{|G(z)|} \leq c\|y\|_{B\{z\}} .
$$

Therefore $G(\cdot) T \mathscr{G}(\mathscr{A})$ is a $z$-Calderón subspace with constant $c$ for all $z$ such that $|z-s|<\delta$.

It is also clear that

$$
(G(\cdot) T \mathscr{G}(A))_{\{z\}}=T_{z} A\{z\} .
$$

Thus, by Theorem 2.6,

$$
\begin{aligned}
\operatorname{codim} T_{z} A\{z\} & =\operatorname{codim}(G(\cdot) T \mathscr{G}(\mathscr{A}))_{\{z\}} \\
& =\operatorname{codim}(G(\cdot) T \mathscr{G}(\mathscr{A}))_{\{s\}} \\
& =\operatorname{codim} T_{s} A\{s\} ;
\end{aligned}
$$

$\operatorname{ker} T_{z}=\{0\}$, and the theorem is proved.

Corollary 2.8. If $\operatorname{dim} \operatorname{ker} T_{s}=d<\infty$ and $T_{s}$ is surjective, then there exists $\delta>0$, so that for all $|z-s|<\delta, \operatorname{dim} \operatorname{ker} T_{z}=d<\infty$ and $T_{s}$ is surjective.

Proof. Consider $T^{*}: B^{*}(\gamma) \rightarrow A^{*}(\gamma)$. Clearly $\operatorname{codim} T_{s}^{*}=\operatorname{dim} \operatorname{ker} T_{s}=d<$ $\infty, \operatorname{dim} \operatorname{ker} T_{s}^{*}=\operatorname{codim} T_{s}=0$ and $\left\|T^{*}\right\|_{B^{*}(\gamma) \rightarrow A^{*}(\gamma)}=\|T\|_{A(\gamma) \rightarrow B(\gamma)}$. By assumption, $\left\{A_{\gamma}^{*}\right\},\left\{B_{\gamma}^{*}\right\}$ are interpolation families and $(A\{z\})^{*}=\left\{A_{\gamma}^{*}\right\}\{z\}$, $(B\{z\})^{*}=\left\{B_{\gamma}^{*}\right\}\{z\}$.

By Theorem 2.7, there exists $\delta>0$ so that $|z-s|<\delta$ implies $\operatorname{ker} T_{z}^{*}=\{0\}$ and $\operatorname{codim} T_{z}^{*}=d$. Therefore, for all these $z$ we have $\operatorname{dim} \operatorname{ker} T_{z}=d$ and $T_{z}$ is surjective.

\section{REFERENCES}

1. E. Albrecht, Spectral interpolation, Oper. Theory: Adv. Appl. 14 (1984), 13-37.

2. J. Bergh and J. Löfström, Interpolation spaces, Springer-Verlag, New York and Berlin, 1976.

3. A. P. Calderón, Intermediate spaces and interpolation, the complex method, Studia Math. 24 (1964), 113-190.

4. W. Cao and Y. Sagher, Stability of Fredholm properties in interpolation scales, Ark. für Mat. (to appear). 
5. R. Coifman, M. Cwikel, R. Rochberg, Y. Sagher, and G. Weiss, The complex method for interpolation of operators acting on families of Banach spaces, Lecture Notes in Math. vol. 779, Springer-Verlag, Berlin, Heidelberg, and New York, 1980, 123-153.

6. _ A theory of complex interpolation for families of Banach spaces, Adv. in Math. 33 (1982), 203-229.

7. P. L. Duren, Theory of $H^{p}$ spaces, Academic Press, New York and London, 1970.

8. J. Lindenstraus and L. Tzafriri, Classical Banach spaces, Springer-Verlag, Berlin, Heidelberg and New York, 1977.

9. I. Ya. Sneiberg, Spectral properties of linear operators in interpolation families of Banach spaces, Mat. Issled. 9 (1974), 214-229.

10. G. Szegö, Über die Randwerte einer analytischen Funktion, Math. Ann. 84 (1921), 482-492.

11. A. T. Vignati and M. Vignati, Spectral theory and complex interpolation, J. Funct. Anal. 80 (1988), 383-397.

12. M. Zafran, Spectral theory and interpolation of operators, J. Funct. Anal. 36 (1980), 185204.

Department of Mathematics, University of Illinois at Chicago, Chicago, Illinois 60680 